\title{
Respuestas Cochrane a problemas comunes
}

\author{
Cochrane Answers to Common Medical Problems
}

Agustìn Ciapponi*

Esta sección tiene por objeto responder a problemas comunes de la práctica ambulatoria mediante evidencia científica de alta calidad como son las Revisiones Cochrane.

EI Nuevo Portal Cochrane BVS http://cochrane.bvsalud.org/portal/php/index.php permite el acceso a las versiones en inglés y en español (con un trimestre de latencia requerido para su traducción). Presentamos resúmenes de pocas

líneas que describen el problema, formulamos la pregunta y brindamos la respuesta Cochrane pertinente

\section{- Parálisis facial idiopática}

La parálisis facial de causa desconocida ("parálisis de Bell") es un trastorno agudo del nervio facial, que produce la pérdida del movimiento en la hemicara. La parálisis mejora generalmente sin tratamiento.

Las terapias físicas, como el ejercicio, la electroestimulación, el láser, la electro o termoterapia y los masajes son usados para acelerar la recuperación, no siendo claro su beneficio.

¿Cuál es la eficacia de la fisioterapia para el tratamiento de la parálisis facial idiopática?

Respuesta Cochrane (revisión sistemática de Julio de 2008 que incluyó seis ensayos clínicos y 547 pacientes con parálisis de Bell) ${ }^{1}$

Las intervenciones evaluadas fueron la electroestimulación (tres trabajos con un total de 294 participantes) y los ejercicios (tres estudios con un total de 253 participantes). Desde el punto de vista clínico, no se registraron beneficios ni daños significativos, cuando se comparó entre sí a las terapias descriptas previamente o contra la ausencia de tratamiento.

Existe limitada evidencia de que las mejorías serían de inicio más rápido con ejercicios físicos, lo que deberá ser confirmado en ensayos aleatorizados y controlados de calidad.

1. Teixeira Lázaro Juliano, Soares Bernardo Garcia de Oliveira, Vieira Vanessa Pedrosa, Prado Gilmar F. Physical therapy for Bell's palsy (idiopathic facial paralysis) (Cochrane Review). In: The Cochrane Library, Issue 4, 2008. Oxford: Update Software. Disponible en: http://cochrane.bvsalud.org/cochrane/show.php?db=reviews\&mfn=3427\& id=\&lang=es\&dblang=\&lib=COC\&print=yes (La versión en español no está aun disponible) PMID: 18646144.

\section{- Otitis media aguda recurrente (OMAR) en niños}

La otitis media aguda es una causa habitual de morbilidad en niños. Se estableció que existe recurrencia cuando un niño sufre tres o más episodios agudos durante el lapso de seis meses o un mínimo de cuatro en un año. Las estrategias terapéuticas incluyen la valoración y modificación de los factores de riesgo, la prescripción de antibióticos ante cada nueva infección, la profilaxis antibiótica y la inserción de tubos de ventilación (diábolos).

¿Cuál es la eficacia de la inserción de diábolos para limitar las recurrencias de la OMAR en la infancia?

Respuesta Cochrane (revisión sistemática de Octubre de 2008 que incluyó dos ensayos aleatorizados que incluyeron en total 148 niños de hasta tres años) ${ }^{2}$

EI NNT para prevenir más de un episodio en seis meses fue de tres (IC95\% 2 a 5$)$.

En un estudio que había incluido 95 niños la inserción de tubos permitió una reducción media de 1,5 episodios durante los primeros seis meses de tratamiento y una significativa $(p<$ 0,001 ) mayor cantidad de niños sin episodios.

El segundo estudio, que había incluido 53 niños, mostró una reducción no significativa de los episodios durante los primeros seis meses, $y$ un incremento no significativo $(p=0,16)$ de niños sin episodios.

Los diábolos tienen un papel importante en mantener a los niños libres de enfermedad durante los primeros seis meses después de la inserción.

Se requieren nuevas investigaciones para determinar su eficacia y sus posibles efectos adversos, más allá de los seis meses iniciales.

2. McDonald Stephen, Langton Hewer Claire D, Nunez Desmond A Grommets (ventilation tubes) for recurrent acute otitis media in children (Cochrane Review). In: The Cochrane Library, Issue 4, 2008. Oxford: Update Software. Disponible en:

http://cochrane.bvsalud.org/cochrane/show.php?db=reviews\&mfn=2706 \&id=\&lang=es\&dblang=\&lib=COC\&print=yes\# $($ La versión en español no está aun disponible). PMID: 18843668. 